\title{
Prognostic impact of atherogenic index of plasma (AIP) in type 2 diabetes mellitus patients with acute coronary syndrome undergoing percutaneous coronary intervention
}

\section{Xiaoteng Ma}

Beijing An Zhen Hospital: Capital Medical University Affiliated Anzhen Hospital

\section{Yan Sun}

Beijing An Zhen Hospital: Capital Medical University Affiliated Anzhen Hospital

\section{Yujing Cheng}

Beijing An Zhen Hospital: Capital Medical University Affiliated Anzhen Hospital Hua Shen

Beijing An Zhen Hospital: Capital Medical University Affiliated Anzhen Hospital

\section{Fei Gao}

Beijing An Zhen Hospital: Capital Medical University Affiliated Anzhen Hospital Jing Qi

Beijing An Zhen Hospital: Capital Medical University Affiliated Anzhen Hospital

\section{Lixia Yang}

Beijing An Zhen Hospital: Capital Medical University Affiliated Anzhen Hospital

\section{Zhijian Wang}

Beijing An Zhen Hospital: Capital Medical University Affiliated Anzhen Hospital

\section{Dongmei Shi}

Beijing An Zhen Hospital: Capital Medical University Affiliated Anzhen Hospital

\section{Yuyang Liu}

Beijing An Zhen Hospital: Capital Medical University Affiliated Anzhen Hospital

Xiaoli Liu ( $D$ liux19881@163.com)

Capital Medical University Affiliated Anzhen Hospital

\section{Yujie Zhou}

Beijing An Zhen Hospital: Capital Medical University Affiliated Anzhen Hospital

\section{Original investigation}

Keywords: Atherogenic index of plasma, Type 2 diabetes mellitus, Acute coronary syndrome, Percutaneous coronary intervention, Adverse cardiovascular events 
Posted Date: September 14th, 2020

DOl: https://doi.org/10.21203/rs.3.rs-67794/v1

License: (c) (i) This work is licensed under a Creative Commons Attribution 4.0 International License. Read Full License 


\section{Abstract}

Background and purposes

The association of atherogenic index of plasma (AIP), an emerging lipid index which can predict risk for cardiovascular (CV) disease, with adverse outcomes in type 2 diabetes mellitus (T2DM) patients with acute coronary syndrome (ACS) undergoing percutaneous coronary intervention ( $\mathrm{PCl}$ ) has been undetermined. Therefore, the aim of this study was to investigate whether AIP could independently predict adverse $\mathrm{CV}$ events in T2DM patients with ACS undergoing PCl.

\section{Methods}

This study was a retrospective analysis of a single centre prospective registry involving 826 consecutive T2DM patients who underwent coronary angiography for ACS and were treated with primary or elective $\mathrm{PCl}$ at our CV center from June 2016 to November 2017. This study eventually included 798 patients (age, $61 \pm 10$ years, male, $72.7 \%$ ). AlP was calculated as the base 10 logarithm of the ratio of plasma concentration of triglycerides to high density lipoprotein-cholesterol (HDL-C). All patients were divided into 4 groups based on the AIP quartiles. The primary endpoint was a composite of all-cause death, nonfatal ischemic stroke, non-fatal myocardial infarction (MI), or unplanned repeat revascularization. The key secondary endpoint was a composite of cardiovascular death, non-fatal ischemic stroke, or non-fatal Ml.

Results

During a median follow-up period of 927 days, 198 patients developed at least one event. Unadjusted Kaplan-Meier analysis showed the incidence of the primary endpoint increased gradually with rising AIP quartiles (log-rank test, $P=0.001$ ). Adjusted multivariate Cox proportional hazards analyses revealed that compared with the lowest AIP quartile, the top AIP quartile was associated with significantly increased risk for the primary and key secondary endpoints (hazard ratio [HR]: $2.153 ; 95 \%$ confidence interval [Cl]: 1.355 to $3.421 ; P=0.001$, and HR: $2.613 ; 95 \% \mathrm{Cl}: 1.024$ to $6.666 ; P=0.044$, respectively). Inclusion of AIP quartiles in a baseline prediction model for the primary endpoint increased the Harrell's C statistic from 0.697 to 0.707 . More importantly, addition of AIP quartiles to the above model significantly improved the continuous net reclassification improvement (continuous NRI $=19.1 \%, P<0.001$ ).

Conclusion

A higher AIP value on admission was independently and strongly associated with adverse CV events in T2DM patients with ACS undergoing PCl.

\section{Background}

Type 2 diabetes mellitus (T2DM) has been established as an important risk factor for adverse outcomes in patients with acute coronary syndrome (ACS) [1-3]; this may be due to a rapid development of atherosclerosis related to T2DM [4]. T2DM patients with ACS remain at a very high residual risk of 
adverse cardiovascular (CV) events and mortality, despite receiving percutaneous coronary intervention (PCI) and guideline-recommended first-line medication therapies [5-8]. Therefore, further risk assessment of such patients may help better determine prognosis and guide better medical management.

Dyslipidemia is a common comorbidity in T2DM and ACS patients. The dyslipidemic pattern in diabetic patients [mainly characterized by high levels of fasting and postprandial triglyceride-rich lipoproteins, reduced high density lipoprotein-cholesterol (HDL-C), normal or slightly elevated low density lipoproteincholesterol (LDL-C), and the appearance of small dense LDL-C particles] is somewhat different from that in non-diabetic patients [9]. Atherogenic index of plasma (AIP), readily calculated from the lipid profile as $\log _{10}$ (triglycerides/HDL-C), is considered an alternative and simple marker of plasma atherogenicity based on an observed significant, positive relationship between AIP and cholesterol esterification rates in apoB-lipoprotein-depleted plasma (FER(HDL)), very low density lipoprotein particle size, remnant lipoprotein particle cholesterol, and LDL density, and inverse correlation of AIP with particle sizes of HDL and LDL [10-16]. As a consequence, we presume that AIP can better reflect the lipid characteristics of diabetic patients. Recently, AIP has been demonstrated to be associated with CV morbidity and mortality in general population and different patients groups [17-28]. However, the prognostic impact of AIP on admission among T2DM patients with ACS undergoing $\mathrm{PCl}$ has not been exclusively studied. We hypothesized that AIP on admission is an independent predictor of adverse CV events in T2DM and ACS patients who underwent $\mathrm{PCl}$.

\section{Methods}

\section{Study population}

This study was a retrospective analysis of a single centre prospective registry involving 826 consecutive T2DM patients who underwent coronary angiography for ACS and were treated with primary or elective $\mathrm{PCl}$ at our CV center from June 2016 to November 2017 [29]. For purposes of this study, we excluded patients with prior coronary artery bypass graft surgery, cardiogenic shock, left ventricular ejection fraction (LVEF) $<30 \%$, and severe renal impairment requiring hemodialysis. Three patients were also excluded because of missing follow-up data despite at least 4 separate attempts to contact them. Finally, 798 patients were included in the present analysis. None of these patients were treated with drugs specifically designed to lower triglycerides or raise HDL-C, such as fibrates, niacin, and omega-3 fatty acids, before admission and at discharge.

\section{Measurement}

Data on demographics, personal medical history, and medication history were collected using a standard questionnaire. The levels of plasma triglycerides, total cholesterol (TC), highdensity lipoprotein cholesterol (HDL-C), and glucose in the first fasting blood samples during the stay in the hospital, which were obtained after $12 \mathrm{~h}$ of fasting, were determined at the central laboratory of Beijing Anzhen Hospital. The low-density lipoprotein cholesterol (LDL-C) level was calculated using the 
Friedewald equation. AIP was calculated as the base 10 logarithm of the ratio of plasma concentration of triglycerides to high density lipoprotein-cholesterol (HDL-C) [10].

\section{Follow-up and endpoints}

All patients were followed up at 1 month and then every 6 months after hospital discharge. Trained personnel who were blinded to the baseline data of patients obtained information on adverse events via telephone contact with patients or their family members using a standardized questionnaire; the adverse events were then ascertained from a careful review of corresponding medical records. The primary endpoint was a composite of all-cause death, non-fatal ischemic stroke, non-fatal myocardial infarction (MI), or unplanned repeat revascularization. The key secondary endpoint was a composite of cardiovascular death, non-fatal ischemic stroke, or non-fatal MI. Adverse events were defined in accordance with our previous publications $[29,30]$. The most severe endpoint event was selected for the primary endpoint analysis if $>1$ event occurred during follow-up (death $>$ stroke $>\mathrm{Ml}>$ revascularization). If more than one stroke or $\mathrm{Ml}$ or revascularization occurred, the first stroke or Ml or revascularization was selected. The follow-up period of the present study lasted until November 2019.

\section{Statistical analysis}

All patients were stratified into 4 groups (Q1 [AIP $\leq 0.0147$ ], Q2 [0.0147< AIP $\leq 0.1850$ ], Q3 [0.1850< AIP $\leq 0.3517$ ] and Q4 [AIP >0.3517]) according to the AIP quartiles. Continuous variables with parametric distributions are shown as the means \pm standard deviations and those with nonparametric distributions are shown as medians and interquartile ranges. Categorical variables are shown as frequencies and percentages. Unpaired t-test or Mann-Whitney $\mathrm{U}$ test and Analysis of Variance or Kruskal-Wallis $\mathrm{H}$ test were used to assess the statistical significance of differences for continuous variables between groups. The statistical significance of differences for categorical variables between groups was analyzed using chi-squared test or Fisher's exact test. Survival analyses of the primary and key second endpoints were performed using Kaplan-Meier curves and Cox proportional hazards model. Differences among KaplanMeier estimates were evaluated with the log-rank test. Results of Cox proportional hazards analyses were presented as hazard ratios (HRs) with $95 \%$ confidence intervals (Cls). Variables with statistical significance and those with clinical importance were entered into the multivariate regression model. Posthoc subgroup analyses stratified by age ( $<60$ versus $\geq 60$ years), sex (female versus male), body mass index (BMI) ( $<28$ versus $\geq 28 \mathrm{~kg} / \mathrm{m}^{2}$ ), LDL-C ( $\leq 1.8$ versus $>1.8 \mathrm{mmol} / \mathrm{L}$ ), hypertension (yes versus no), and clinical presentation (non-ST versus ST segment elevation ACS) were employed to examine the consistence of the prognostic significance of AIP for the primary endpoint. Harrell's C statistic for the primary endpoint was calculated after the addition of AIP quartiles to other independent predictors identified in the multivariate Cox proportional hazards analyses; it is generally considered Harrell's C statistic above 0.7 acceptable discriminatory power, that above 0.8 excellent discriminatory power, and that above 0.9 outstanding discriminatory power. The incremental effect of adding AIP quartiles to other independent predictors in predicting the primary endpoint was evaluated using the continuous net reclassification improvement (NRI). Analyses were performed using SPSS version 24.0 (IBM Corp., 
Armonk, New York, US) and R software version 3.5.3 (R Foundation for Statistical Computing, Beijing, China). A 2-sided $P$-value $<0.05$ was considered significant.

\section{Results}

A total of 798 T2DM and ACS patients who underwent PCl were included in the present study. The mean age of these patients was $61 \pm 10$ years, and $72.7 \%$ of patients were men. The baseline clinical and laboratory characteristics of the study patients according to the AIP quartiles are listed in Table 1. Patients with higher AIP values tended to be younger, were predominantly male, had higher rates of current smoking and dyslipidaemia, and had lower rates of never smoking and diagnosis with unstable angina pectoris. Patients with higher AIP values were more likely to have higher levels of BMI, serum creatinine (SCr), uric acid, TC, LDL-C, triglycerides, and fasting plasma glucose (FPG), while have lower levels of pulse pressure (PP) and HDL-C. Use of medications, agiographic findings, and procedural results of the study patients according to the AIP quartiles are summarized in Table 2. Medications before admission, intraoperative anticoagulants, and periprocedural medications, except for $\beta$-blockers, did not differ among the different AIP groups. Medications at discharge, except for P2Y12 inhibitors, angiotensin converting enzyme inhibitors/angiotensin II receptor blockers (ACEls/ARBs), and oral antidiabetic agents, were similar across the different AIP groups. More patients who had higher AIP values were treated with ACEIs/ARBs at discharge. The proportions of left main/three-vessel disease and two-vessel disease were different among the different AIP groups. Patients with higher AIP values tended to have a higher rate of chronic total occlusions, and a lower rate of heavy calcification lesions. The proportions of left circumflex artery and right coronary artery interventions were different among the different AIP groups. Patients with higher AIP values tended to have a lower rate of complete revascularization. 
Table 1

Baseline clinical and laboratory characteristics of the study patients according to the AIP quartiles

\begin{tabular}{|c|c|c|c|c|c|}
\hline \multirow[t]{2}{*}{ Variable } & \multirow{2}{*}{$\begin{array}{l}\text { Q1 } \\
n=199\end{array}$} & \multirow{2}{*}{$\begin{array}{l}\text { Q2 } \\
n=200\end{array}$} & \multirow{2}{*}{$\begin{array}{l}\text { Q3 } \\
n=200\end{array}$} & \multirow{2}{*}{$\begin{array}{l}\text { Q4 } \\
n=199\end{array}$} & \multirow[t]{2}{*}{$\begin{array}{l}P \\
\text { value }\end{array}$} \\
\hline & & & & & \\
\hline \multicolumn{6}{|l|}{ Demographics } \\
\hline Age (years) & $63 \pm 8$ & $63 \pm 10$ & $61 \pm 9$ & $58 \pm 12$ & $\begin{array}{l}< \\
0.001\end{array}$ \\
\hline Male sex, n (\%) & $125(62.8)$ & $146(73.0)$ & $153(76.5)$ & $156(78.4)$ & 0.002 \\
\hline \multicolumn{6}{|c|}{$\begin{array}{l}\text { Clinical values (on } \\
\text { admission) }\end{array}$} \\
\hline BMI $\left(\mathrm{kg} / \mathrm{m}^{2}\right)$ & $25.3 \pm 3.1$ & $25.4 \pm 2.8$ & $26.1 \pm 3.1$ & $27.0 \pm 3.5$ & $\stackrel{<}{0.001}$ \\
\hline SBP (mm Hg) & $133 \pm 18$ & $133 \pm 17$ & $130 \pm 16$ & $131 \pm 16$ & 0.116 \\
\hline DBP (mm Hg) & $75 \pm 11$ & $76 \pm 10$ & $75 \pm 10$ & $77 \pm 11$ & 0.203 \\
\hline $\mathrm{PP}(\mathrm{mm} \mathrm{Hg})$ & $58 \pm 16$ & $58 \pm 15$ & $55 \pm 15$ & $54 \pm 14$ & 0.015 \\
\hline
\end{tabular}

\section{Risk factors}

Smoking status

\begin{tabular}{|c|c|c|c|c|c|}
\hline Current smoking, n (\%) & $60(30.2)$ & $77(38.5)$ & $76(38.0)$ & $111(55.8)$ & $\begin{array}{l}<.001 \\
0.00\end{array}$ \\
\hline Former smoking, n (\%) & $33(16.6)$ & $33(16.5)$ & $38(19.0)$ & $26(13.1)$ & 0.455 \\
\hline Never smoking, n (\%) & $106(53.3)$ & $90(45.0)$ & $86(43.0)$ & $62(31.2)$ & $\begin{array}{l}< \\
0.001\end{array}$ \\
\hline $\begin{array}{l}\text { Chronically daily drinking, } \\
\mathrm{n}(\%)\end{array}$ & $14(7.0)$ & $18(9.0)$ & $26(13.0)$ & $21(10.6)$ & 0.234 \\
\hline $\begin{array}{l}\text { Family history of } \mathrm{CHD}, \mathrm{n} \\
(\%)\end{array}$ & $54(27.1)$ & $60(30.0)$ & $69(34.5)$ & $56(28.1)$ & 0.386 \\
\hline Hypertension, n (\%) & $138(69.3)$ & $137(68.5)$ & $141(70.5)$ & $130(65.3)$ & 0.715 \\
\hline Dyslipidaemia, n (\%) & $107(53.8)$ & $174(87.0)$ & $192(96.0)$ & $195(98.0)$ & $\dot{0} 001$ \\
\hline Previous MI, n (\%) & $36(18.1)$ & $43(21.5)$ & $40(20.0)$ & $51(25.6)$ & 0.301 \\
\hline
\end{tabular}

AIP indicates atherogenic index of plasma; BMI, body mass index; SBP, systolic blood pressure; DBP, diastolic blood pressure; PP, pulse pressure; $\mathrm{CHD}$, coronary heart disease; MI, myocardial infarction; $\mathrm{PCl}$, percutaneous coronary intervention; TIA, transient ischemic attack; PAD, peripheral artery disease; LVEF, left ventricular ejection fraction; UAP, unstable angina pectoris; NSTEMI, non ST-segment elevation myocardial infarction; STEMI, ST-segment elevation myocardial infarction; BUN, blood urea nitrogen; SCr, serum creatinine; UA, uric acid; TC, total cholesterol; LDL-C, low-density lipoproteincholesterol; HDL-C, high-density lipoprotein-cholesterol; FPG, fasting plasma glucose. 


\begin{tabular}{|c|c|c|c|c|c|}
\hline Variable & $\begin{array}{l}\text { Q1 } \\
n=199\end{array}$ & $\begin{array}{l}\text { Q2 } \\
n=200\end{array}$ & $\begin{array}{l}Q 3 \\
n=200\end{array}$ & $\begin{array}{l}\text { Q4 } \\
n=199\end{array}$ & $\begin{array}{l}P \\
\text { value }\end{array}$ \\
\hline Past PCl, n (\%) & $47(23.6)$ & $50(25.0)$ & $50(25.0)$ & $44(22.1)$ & 0.891 \\
\hline $\begin{array}{l}\text { Previous ischemic stroke } \\
\text { or TIA, } n(\%)\end{array}$ & $13(6.5)$ & $13(6.5)$ & $17(8.5)$ & $8(4.0)$ & 0.338 \\
\hline PAD, n (\%) & $27(13.6)$ & $29(14.5)$ & $31(15.5)$ & $35(17.6)$ & 0.713 \\
\hline Cardiac failure, $\mathrm{n}(\%)$ & $12(6.0)$ & $13(6.5)$ & $23(11.5)$ & $19(9.5)$ & 0.156 \\
\hline LVEF (\%) & $64(60-67)$ & $64(60-68)$ & $65(60-68)$ & $65(59-68)$ & 0.963 \\
\hline \multicolumn{6}{|l|}{ Clinical presentation } \\
\hline UAP, n (\%) & $171(85.9)$ & $153(76.5)$ & $150(75.0)$ & $153(76.9)$ & 0.033 \\
\hline NSTEMI, n (\%) & $19(9.5)$ & $28(14.0)$ & $28(14.5)$ & $25(12.6)$ & 0.493 \\
\hline STEMI, n (\%) & $9(4.5)$ & $19(9.5)$ & $22(11.0)$ & $21(10.6)$ & 0.088 \\
\hline \multicolumn{6}{|l|}{$\begin{array}{l}\text { Laboratory measurements } \\
\text { (fasting state) }\end{array}$} \\
\hline BUN (mmol/L) & $\begin{array}{l}5.2(4.3- \\
6.3)\end{array}$ & $5.4(4.5-6.5)$ & $5.4(4.6-6.2)$ & $\begin{array}{l}5.5(4.6- \\
6.7)\end{array}$ & 0.173 \\
\hline $\mathrm{SCr}(\mu \mathrm{mol} / \mathrm{L})$ & $\begin{array}{l}66.5(59.3- \\
73.6)\end{array}$ & $\begin{array}{l}69.2(61.9- \\
80.3)\end{array}$ & $\begin{array}{l}70.3(62.2- \\
81.3)^{(6-}\end{array}$ & $\begin{array}{l}71.5(63.2- \\
82.2)\end{array}$ & $\begin{array}{l}< \\
0.001\end{array}$ \\
\hline $\mathrm{UA}(\mu \mathrm{mol} / \mathrm{L})$ & $\begin{array}{l}303.2 \\
(271.7- \\
347.2)\end{array}$ & $\begin{array}{l}321.3 \\
(279.6- \\
373.0)\end{array}$ & $\begin{array}{l}323.8 \\
(282.5- \\
386.0)\end{array}$ & $\begin{array}{l}368.5 \\
(307.0- \\
410.3)\end{array}$ & $\begin{array}{l}< \\
0.001\end{array}$ \\
\hline $\mathrm{TC}(\mathrm{mmol} / \mathrm{L})$ & $3.90 \pm 0.96$ & $4.06 \pm 1.01$ & $4.10 \pm 0.99$ & $4.47 \pm 1.03$ & ¿ 0.001 \\
\hline LDL-C (mmol/L) & $2.23 \pm 0.85$ & $2.48 \pm 0.84$ & $2.48 \pm 0.77$ & $2.53 \pm 0.76$ & 0.001 \\
\hline $\mathrm{HDL}-\mathrm{C}(\mathrm{mmol} / \mathrm{L})$ & $1.23 \pm 0.22$ & $1.04 \pm 0.17$ & $0.95 \pm 0.17$ & $0.87 \pm 0.14$ & $\begin{array}{l}< \\
0.001\end{array}$ \\
\hline Triglycerides (mg/dl) & $\begin{array}{l}0.88(0.72- \\
1.00)\end{array}$ & $\begin{array}{l}1.31(1.18- \\
1.48)\end{array}$ & $\begin{array}{l}1.71(1.51- \\
1.99)\end{array}$ & $\begin{array}{l}2.66(2.25- \\
3.38)\end{array}$ & ¿ 0.001 \\
\hline FPG $(\mathrm{mg} / \mathrm{dl})$ & $\begin{array}{l}6.80(6.12- \\
8.23)\end{array}$ & $\begin{array}{l}7.17(6.32- \\
8.25)\end{array}$ & $\begin{array}{l}6.80(5.91- \\
7.88)\end{array}$ & $\begin{array}{l}7.65(6.61- \\
8.49)\end{array}$ & $\begin{array}{l}<.001 \\
0.001\end{array}$ \\
\hline
\end{tabular}

AIP indicates atherogenic index of plasma; BMI, body mass index; SBP, systolic blood pressure; DBP, diastolic blood pressure; PP, pulse pressure; $\mathrm{CHD}$, coronary heart disease; MI, myocardial infarction; $\mathrm{PCl}$, percutaneous coronary intervention; TIA, transient ischemic attack; PAD, peripheral artery disease; LVEF, left ventricular ejection fraction; UAP, unstable angina pectoris; NSTEMI, non ST-segment elevation myocardial infarction; STEMI, ST-segment elevation myocardial infarction; BUN, blood urea nitrogen; SCr, serum creatinine; UA, uric acid; TC, total cholesterol; LDL-C, low-density lipoproteincholesterol; HDL-C, high-density lipoprotein-cholesterol; FPG, fasting plasma glucose. 


\begin{tabular}{|c|c|c|c|c|c|}
\hline \multirow[t]{2}{*}{ Variable } & Q1 & & & & \multirow{2}{*}{$\begin{array}{l}P \\
\text { value }\end{array}$} \\
\hline & $n=199$ & $n=200$ & $n=200$ & $n=199$ & \\
\hline Glycated haemoglobin (\%) & $\begin{array}{l}7.0(6.6- \\
8.1)\end{array}$ & $7.3(6.7-8.2)$ & $7.2(6.6-8.1)$ & $7.3(6.7-8.0)$ & 0.409 \\
\hline AIP & $\begin{array}{l}-0.1581 \pm \\
0.1373\end{array}$ & $\begin{array}{l}0.1029 \pm \\
0.0482\end{array}$ & $\begin{array}{l}0.2631 \pm \\
0.0504\end{array}$ & $\begin{array}{l}0.5272 \pm \\
0.1773\end{array}$ & $<.001$ \\
\hline \multicolumn{6}{|c|}{$\begin{array}{l}\text { AIP indicates atherogenic index of plasma; BMI, body mass index; SBP, systolic blood pressure; DBP, } \\
\text { diastolic blood pressure; PP, pulse pressure; CHD, coronary heart disease; MI, myocardial infarction; } \\
\text { PCI, percutaneous coronary intervention; TIA, transient ischemic attack; PAD, peripheral artery disease; } \\
\text { LVEF, left ventricular ejection fraction; UAP, unstable angina pectoris; NSTEMl, non ST-segment } \\
\text { elevation myocardial infarction; STEMI, ST-segment elevation myocardial infarction; BUN, blood urea } \\
\text { nitrogen; SCr, serum creatinine; UA, uric acid; TC, total cholesterol; LDL-C, low-density lipoprotein- } \\
\text { cholesterol; HDL-C, high-density lipoprotein-cholesterol; FPG, fasting plasma glucose. }\end{array}$} \\
\hline
\end{tabular}


Table 2

Use of medications, agiographic findings, and procedural results of the study patients according to the AlP quartiles

\begin{tabular}{|c|c|c|c|c|c|}
\hline Variable & $\begin{array}{l}\text { Q1 } \\
n=199\end{array}$ & $\begin{array}{l}\text { Q2 } \\
n=200\end{array}$ & $\begin{array}{l}\text { Q3 } \\
n=200\end{array}$ & $\begin{array}{l}\text { Q4 } \\
n=199\end{array}$ & $\begin{array}{l}P \\
\text { value }\end{array}$ \\
\hline \multicolumn{6}{|l|}{ Medications before admission } \\
\hline Aspirin, n (\%) & $145(72.9)$ & $149(74.5)$ & $154(77.0)$ & $146(73.4)$ & 0.784 \\
\hline P2Y12 inhibitors, n (\%) & $81(40.7)$ & $81(40.5)$ & $74(37.0)$ & $80(40.2)$ & 0.858 \\
\hline Statins, n (\%) & $150(75.4)$ & $143(71.5)$ & $155(77.5)$ & $142(71.4)$ & 0.418 \\
\hline ACEIs/ARBs, n (\%) & $55(27.6)$ & $65(32.5)$ & $72(36.0)$ & $69(34.7)$ & 0.300 \\
\hline ß-blockers, n (\%) & $70(35.2)$ & 79 (39.5) & $93(46.5)$ & $63(31.7)$ & 0.016 \\
\hline Insulin, n (\%) & $75(37.7)$ & $73(36.5)$ & 79 (39.5) & $63(31.7)$ & 0.406 \\
\hline Oral antidiabetic agents, $\mathrm{n}(\%)$ & $89(44.7)$ & $98(49.0)$ & $110(55.0)$ & $90(45.2)$ & 0.145 \\
\hline \multicolumn{6}{|l|}{ Intraoperative anticoagulants } \\
\hline Unfractionated heparin, n (\%) & $168(84.4)$ & $170(85.0)$ & $154(77.0)$ & $156(78.4)$ & 0.086 \\
\hline LMWH, n (\%) & $6(3.0)$ & $7(3.5)$ & $16(8.0)$ & $10(5.0)$ & 0.089 \\
\hline Bivalirudin, n (\%) & $25(12.6)$ & $23(11.5)$ & $30(15.0)$ & $33(16.6)$ & 0.448 \\
\hline \multicolumn{6}{|l|}{ Perioperative medications } \\
\hline Aspirin, n (\%) & $196(98.5)$ & $198(99.0)$ & $\begin{array}{l}200 \\
(100.0)\end{array}$ & $196(98.5)$ & 0.308 \\
\hline P2Y12 inhibitors, n (\%) & $\begin{array}{l}199 \\
(100.0)\end{array}$ & $\begin{array}{l}200 \\
(100.0)\end{array}$ & $\begin{array}{l}200 \\
(100.0)\end{array}$ & $\begin{array}{l}199 \\
(100.0)\end{array}$ & - \\
\hline $\mathrm{GP} \otimes \mathrm{b} /$ \a receptor antagonist, $\mathrm{n}(\%)$ & $29(14.6)$ & $38(19.0)$ & $45(22.5)$ & $37(18.6)$ & 0.246 \\
\hline \multicolumn{6}{|l|}{ Medications at discharge } \\
\hline Aspirin, n (\%) & $196(98.5)$ & $198(99.0)$ & $\begin{array}{l}200 \\
(100.0)\end{array}$ & $196(98.5)$ & 0.308 \\
\hline Cilostazol, n (\%) & $3(1.5)$ & $2(1.0)$ & $1(0.5)$ & $4(2.0)$ & 0.473 \\
\hline Clopidogrel, n (\%) & $174(87.4)$ & $183(91.5)$ & $178(89.0)$ & $190(95.5)$ & 0.031 \\
\hline Ticagrelor, n (\%) & 25 (12.6) & $17(8.5)$ & $22(11.0)$ & $9(4.5)$ & 0.031 \\
\hline
\end{tabular}

AIP indicates atherogenic index of plasma; ACEls, angiotensin converting enzyme inhibitors; ARBs, angiotensin II receptor blockers; LM, left-main artery; LAD, left anterior descending artery; LCX, left circumflex artery; RCA; right coronary artery; DES, drug-eluting stent; BRS, bioresorbable scaffold; DCB, drug-coated balloon. 


\begin{tabular}{|c|c|c|c|c|c|}
\hline \multirow[t]{2}{*}{ Variable } & Q1 & Q2 & & & \multirow{2}{*}{$\begin{array}{l}P \\
\text { value }\end{array}$} \\
\hline & $n=199$ & $n=200$ & $\mathrm{n}=\mathbf{2 0 0}$ & $n=199$ & \\
\hline Statins, n (\%) & $\begin{array}{l}199 \\
(100.0)\end{array}$ & $\begin{array}{l}200 \\
(100.0)\end{array}$ & $\begin{array}{l}200 \\
(100.0)\end{array}$ & $\begin{array}{l}199 \\
(100.0)\end{array}$ & - \\
\hline ACEIs/ARBs, n (\%) & $81(40.7)$ & $93(46.5)$ & $105(52.5)$ & $121(60.8)$ & 0.001 \\
\hline ß-blockers, n (\%) & $138(69.3)$ & $147(73.5)$ & $155(77.5)$ & $136(68.3)$ & 0.155 \\
\hline Insulin, n (\%) & $65(32.7)$ & $76(38.0)$ & $72(36.0)$ & $57(28.6)$ & 0.213 \\
\hline Oral antidiabetic agents, n (\%) & $87(43.7)$ & $120(60.0)$ & $119(59.5)$ & $105(52.8)$ & 0.003 \\
\hline \multicolumn{6}{|l|}{ Angiographic findings } \\
\hline One-vessel disease, n (\%) & $22(11.1)$ & $15(7.5)$ & $18(9.0)$ & $25(12.6)$ & 0.347 \\
\hline Two-vessel disease, n (\%) & $68(34.2)$ & $41(20.5)$ & $42(21.0)$ & $46(23.1)$ & 0.004 \\
\hline LM/three-vessel disease, $n(\%)$ & $109(54.8)$ & $144(72.0)$ & $140(70.0)$ & $128(64.3)$ & 0.001 \\
\hline Proximal LAD stenosis, $\mathrm{n}(\%)$ & $94(47.2)$ & $110(55.0)$ & $97(48.5)$ & $100(50.3)$ & 0.428 \\
\hline Restenotic lesions, n (\%) & $26(13.1)$ & $26(13.0)$ & $30(15.0)$ & $29(14.6)$ & 0.913 \\
\hline $\begin{array}{l}\text { Trifurcation or bifurcation lesions, } n \\
(\%)\end{array}$ & $155(77.9)$ & $162(81.0)$ & $151(75.5)$ & $148(74.4)$ & 0.403 \\
\hline Chronic total occlusions, n (\%) & $36(18.1)$ & $46(23.0)$ & $44(22.0)$ & $57(28.6)$ & 0.093 \\
\hline Thrombus lesions, n (\%) & $7(3.5)$ & $14(7.0)$ & $12(6.0)$ & $12(6.0)$ & 0.478 \\
\hline Heavy calcification lesions, n (\%) & $75(37.7)$ & $69(34.5)$ & $73(36.5)$ & $48(24.1)$ & 0.016 \\
\hline Lesions > 20 mm long, $\mathrm{n}(\%)$ & $108(54.3)$ & $112(56.0)$ & $122(61.0)$ & $117(58.8)$ & 0.537 \\
\hline \multicolumn{6}{|l|}{ Procedural results } \\
\hline \multicolumn{6}{|l|}{ Target vessel territory } \\
\hline LM, n (\%) & $13(6.5)$ & $16(8.0)$ & $9(4.5)$ & $18(9.0)$ & 0.313 \\
\hline LAD, n (\%) & $104(52.3)$ & $97(48.5)$ & $100(50.0)$ & $99(49.7)$ & 0.899 \\
\hline LCX, n (\%) & $64(32.2)$ & $69(34.5)$ & $42(21.0)$ & $48(24.1)$ & 0.007 \\
\hline $\mathrm{RCA}, \mathrm{n}(\%)$ & $69(34.7)$ & $82(41.0)$ & $100(50.0)$ & $74(37.2)$ & 0.011 \\
\hline DES use, n (\%) & $174(87.4)$ & $172(86.0)$ & $161(80.5)$ & $159(79.9)$ & 0.097 \\
\hline
\end{tabular}

AIP indicates atherogenic index of plasma; ACEls, angiotensin converting enzyme inhibitors; ARBs, angiotensin II receptor blockers; LM, left-main artery; LAD, left anterior descending artery; LCX, left circumflex artery; RCA; right coronary artery; DES, drug-eluting stent; BRS, bioresorbable scaffold; DCB, drug-coated balloon. 


\begin{tabular}{|c|c|c|c|c|c|}
\hline \multirow[t]{2}{*}{ Variable } & \multirow{2}{*}{$\begin{array}{l}\text { Q1 } \\
n=199\end{array}$} & \multirow{2}{*}{$\begin{array}{l}\text { Q2 } \\
n=200\end{array}$} & \multirow{2}{*}{$\begin{array}{l}\text { Q3 } \\
n=200\end{array}$} & \multirow{2}{*}{$\begin{array}{l}\text { Q4 } \\
n=199\end{array}$} & \multirow{2}{*}{$\begin{array}{l}P \\
\text { value }\end{array}$} \\
\hline & & & & & \\
\hline BRS use, n (\%) & $6(3.0)$ & $7(3.5)$ & $8(4.0)$ & $11(5.5)$ & 0.607 \\
\hline DCB use, $\mathrm{n}(\%)$ & $12(6.0)$ & $11(5.5)$ & $19(9.5)$ & $14(7.0)$ & 0.409 \\
\hline Complete revascularization, $\mathrm{n}(\%)$ & $136(68.3)$ & $115(57.5)$ & $110(55.0)$ & $107(53.8)$ & 0.012 \\
\hline \multicolumn{6}{|c|}{$\begin{array}{l}\text { AIP indicates atherogenic index of plasma; ACEls, angiotensin converting enzyme inhibitors; ARBs, } \\
\text { angiotensin II receptor blockers; LM, left-main artery; LAD, left anterior descending artery; LCX, left } \\
\text { circumflex artery; RCA; right coronary artery; DES, drug-eluting stent; BRS, bioresorbable scaffold; DCB, } \\
\text { drug-coated balloon. }\end{array}$} \\
\hline
\end{tabular}

The median follow-up duration was 927 days (interquartile range, 774 to 1109 days), and during the follow-up period, 198 patients suffered from at least one adverse CV event, which was recorded in 33 (16.6\%) patients from the Q1 group, 45 (22.5\%) from the Q2 group, 54 (27.0\%) from the Q3 group, and 66 (33.2\%) from the Q4 group. In the 198 patients who had at least one adverse CV event, there were 20 deaths (18 deaths from CV causes and 2 deaths from non-CV causes), 17 cases of non-fatal ischemic stroke, 24 cases of non-fatal $\mathrm{Ml}$, and 180 cases of unplanned revascularization. Of these, 33 patients suffered two, 2 patients suffered three, and 2 patients suffered four adverse CV events. The baseline clinical and laboratory characteristics of the study patients stratified by the primary endpoint are shown in Table 3. Compared with those without events, patients with at least one event had higher levels of AIP. Patients with at least one event had higher rates of previous $\mathrm{MI}$, past $\mathrm{PCl}$, peripheral arterial disease (PAD), and cardiac failure, higher levels of PP, SCr, triglycerides, and FPG, but lower levels of diastolic blood pressure, HDL-C, and LVEF. Use of medications, agiographic findings, and procedural results of the study patients stratified by the primary endpoint are presented in Table 4. Medications before admission, except for ACEIs/ARBs, were not different between patients with and without events. Intraoperative anticoagulants and perioperative medications, except for aspirin, did not differ between patients with and without events. Medications at discharge, except for aspirin, cilostazol, and insulin, were similar between patients with and without events. Compared with those without events, patients with at least one event had higher rates of left main/three-vessel disease, restenotic lesions, and lesions $>20 \mathrm{~mm}$ long, but lower rates of one-vessel and two-vessel diseases. In terms of procedural results, more patients who suffered from at least one event used drug-coated balloon, and fewer patients who suffered from at least one event achieved complete revascularization. 
Table 3

Baseline clinical and laboratory characteristics of the study patients stratified by the primary endpoint

\begin{tabular}{|c|c|c|c|}
\hline Variable & $\begin{array}{l}\text { No such events } \\
n=600\end{array}$ & $\begin{array}{l}\text { Primary endpoint } \\
n=198\end{array}$ & $P$ value \\
\hline \multicolumn{4}{|l|}{ Demographics } \\
\hline Age (years) & $61 \pm 10$ & $62 \pm 10$ & 0.435 \\
\hline Male sex, n (\%) & $436(72.7)$ & $144(72.7)$ & 0.987 \\
\hline \multicolumn{4}{|l|}{ Clinical values (on admission) } \\
\hline BMI $\left(\mathrm{kg} / \mathrm{m}^{2}\right)$ & $26.1 \pm 3.2$ & $25.6 \pm 3.1$ & 0.087 \\
\hline $\mathrm{SBP}(\mathrm{mm} \mathrm{Hg})$ & $131 \pm 17$ & $133 \pm 16$ & 0.399 \\
\hline DBP (mm Hg) & $77 \pm 10$ & $72 \pm 10$ & $<0.001$ \\
\hline PP (mm Hg) & $55 \pm 15$ & $60 \pm 15$ & $<0.001$ \\
\hline \multicolumn{4}{|l|}{ Risk factors } \\
\hline \multicolumn{4}{|l|}{ Smoking status } \\
\hline Current smoking, n (\%) & $244(40.7)$ & $80(40.4)$ & 0.948 \\
\hline Former smoking, n (\%) & $93(15.5)$ & $37(18.7)$ & 0.292 \\
\hline Never smoking, n (\%) & $263(43.8)$ & $81(40.9)$ & 0.471 \\
\hline Chronically daily drinking, n (\%) & $61(10.2)$ & $18(9.1)$ & 0.660 \\
\hline Family history of CHD, n (\%) & $173(28.8)$ & $66(33.3)$ & 0.231 \\
\hline Hypertension, n (\%) & $408(68.0)$ & $138(69.7)$ & 0.656 \\
\hline Dyslipidaemia, n (\%) & $496(82.7)$ & $172(86.9)$ & 0.165 \\
\hline Previous MI, n (\%) & $114(19.0)$ & $56(28.3)$ & 0.006 \\
\hline Past PCI, n (\%) & $123(20.5)$ & $68(34.3)$ & $<0.001$ \\
\hline Previous ischemic stroke or TIA, n (\%) & $39(6.5)$ & $12(6.1)$ & 0.827 \\
\hline PAD, n (\%) & $66(11.0)$ & $56(28.3)$ & $<0.001$ \\
\hline Cardiac failure, $\mathrm{n}(\%)$ & $39(6.5)$ & $28(14.1)$ & 0.001 \\
\hline LVEF (\%) & $65(60-68)$ & $63(57-67)$ & 0.002 \\
\hline
\end{tabular}

Abbreviations as in Tables 1 and 2. 


\begin{tabular}{|c|c|c|c|}
\hline Variable & $\begin{array}{l}\text { No such events } \\
n=600\end{array}$ & $\begin{array}{l}\text { Primary endpoint } \\
n=198\end{array}$ & $P$ value \\
\hline $\begin{array}{l}\text { Clinical presentation } \\
\text { UAP, n (\%) }\end{array}$ & $467(77.8)$ & $160(80.8)$ & 0.376 \\
\hline NSTEMI, n (\%) & $82(13.7)$ & $18(9.1)$ & 0.092 \\
\hline STEMI, n (\%) & $51(8.5)$ & $20(10.1)$ & 0.493 \\
\hline \multicolumn{4}{|c|}{ Laboratory measurements (fasting state) } \\
\hline BUN (mmol/L) & $5.4(4.5-6.3)$ & $5.6(4.5-6.7)$ & 0.179 \\
\hline $\mathrm{SCr}(\mu \mathrm{mol} / \mathrm{L})$ & $68.5(60.9-78.5)$ & $70.7(63.1-83.5)$ & 0.010 \\
\hline $\mathrm{UA}(\mu \mathrm{mol} / \mathrm{L})$ & $333.2 \pm 77.2$ & $344.4 \pm 78.9$ & 0.079 \\
\hline $\mathrm{TC}(\mathrm{mmol} / \mathrm{L})$ & $4.10 \pm 1.03$ & $4.24 \pm 0.98$ & 0.093 \\
\hline LDL-C (mmol/L) & $2.41 \pm 0.83$ & $2.47 \pm 0.74$ & 0.382 \\
\hline $\mathrm{HDL}-\mathrm{C}(\mathrm{mmol} / \mathrm{L})$ & $1.04 \pm 0.22$ & $0.96 \pm 0.20$ & $<0.001$ \\
\hline Triglycerides (mmol/L) & $1.46(1.04-2.00)$ & $1.67(1.12-2.31)$ & 0.003 \\
\hline FPG (mmol/L) & $6.88(6.12-8.01)$ & $7.89(6.70-9.23)$ & $<0.001$ \\
\hline Glycated haemoglobin (\%) & $7.2(6.6-8.1)$ & $7.4(6.8-8.3)$ & 0.079 \\
\hline AIP & $0.1608 \pm 0.2653$ & $0.2535 \pm 0.2919$ & $<0.001$ \\
\hline AIP & & & 0.001 \\
\hline Q1, n (\%) & $166(27.7)$ & $33(16.7)$ & - \\
\hline Q2, n (\%) & $155(25.8)$ & $45(22.7)$ & - \\
\hline Q3, n (\%) & $146(24.3)$ & $54(27.3)$ & - \\
\hline Q4, n (\%) & $133(22.2)$ & 66 (33.3) & - \\
\hline
\end{tabular}


Table 4

Use of medications, agiographic findings, and procedural results of the study patients stratified by the primary endpoint

\begin{tabular}{|c|c|c|c|}
\hline Variable & $\begin{array}{l}\text { No such events } \\
n=600\end{array}$ & $\begin{array}{l}\text { Primary endpoint } \\
n=198\end{array}$ & $P$ value \\
\hline \multicolumn{4}{|l|}{ Medications before admission } \\
\hline Aspirin, n (\%) & $442(73.7)$ & $152(76.8)$ & 0.386 \\
\hline P2Y12 inhibitors, n (\%) & $234(39.0)$ & $82(41.4)$ & 0.547 \\
\hline Statins, n (\%) & $438(73.0)$ & $152(76.8)$ & 0.295 \\
\hline ACEls/ARBs, n (\%) & $183(30.5)$ & $78(39.4)$ & 0.021 \\
\hline$\beta$-blockers, n (\%) & $233(38.8)$ & $72(36.4)$ & 0.535 \\
\hline Insulin, n (\%) & $212(35.3)$ & $78(39.4)$ & 0.303 \\
\hline Oral antidiabetic agents, n (\%) & $289(48.2)$ & $98(49.5)$ & 0.746 \\
\hline \multicolumn{4}{|l|}{ Intraoperative anticoagulants } \\
\hline Unfractionated heparin, n (\%) & $486(81.0)$ & $162(81.8)$ & 0.798 \\
\hline LMWH, n (\%) & $29(4.8)$ & $10(5.1)$ & 0.902 \\
\hline Bivalirudin, n (\%) & $85(14.2)$ & $26(13.1)$ & 0.715 \\
\hline \multicolumn{4}{|l|}{ Perioperative medications } \\
\hline Aspirin, n (\%) & $600(100.0)$ & $190(96.0)$ & $<0.001$ \\
\hline P2Y12 inhibitors, n (\%) & $600(100.0)$ & $198(100.0)$ & - \\
\hline $\mathrm{GP} \rrbracket \mathrm{b} /$ / $\mathrm{a}$ receptor antagonist, $\mathrm{n}(\%)$ & 105 (17.5) & $44(22.2)$ & 0.139 \\
\hline \multicolumn{4}{|l|}{ Medications at discharge } \\
\hline Aspirin, n (\%) & $600(100.0)$ & $190(96.0)$ & $<0.001$ \\
\hline Cilostazol, n (\%) & $2(0.3)$ & $8(4.0)$ & $<0.001$ \\
\hline Clopidogrel, n (\%) & $545(90.8)$ & $180(90.9)$ & 0.974 \\
\hline Ticagrelor, n (\%) & $55(9.2)$ & $18(9.1)$ & 0.974 \\
\hline Statins, n (\%) & $600(100.0)$ & $198(100.0)$ & - \\
\hline ACEls/ARBs, n (\%) & $294(49.0)$ & $106(53.5)$ & 0.268 \\
\hline$\beta$-blockers, n (\%) & $442(73.7)$ & $134(67.7)$ & 0.103 \\
\hline
\end{tabular}




\begin{tabular}{|c|c|c|c|}
\hline Variable & $\begin{array}{l}\text { No such events } \\
n=600\end{array}$ & $\begin{array}{l}\text { Primary endpoint } \\
n=198\end{array}$ & $P$ value \\
\hline Insulin, n (\%) & $190(31.7)$ & $80(40.4)$ & 0.024 \\
\hline Oral antidiabetic agents, $\mathrm{n}(\%)$ & $324(54.0)$ & $106(53.5)$ & 0.909 \\
\hline \multicolumn{4}{|l|}{ Angiographic findings } \\
\hline One-vessel disease, $\mathrm{n}(\%)$ & $72(12.0)$ & $8(4.0)$ & 0.001 \\
\hline Two-vessel disease, n (\%) & $165(27.5)$ & $32(16.2)$ & 0.001 \\
\hline LM/three-vessel disease, $n(\%)$ & $363(60.5)$ & $158(79.8)$ & $<0.001$ \\
\hline Proximal LAD stenosis, $\mathrm{n}(\%)$ & $295(49.2)$ & $106(53.5)$ & 0.286 \\
\hline Restenotic lesions, n (\%) & $62(10.3)$ & $49(24.7)$ & $<0.001$ \\
\hline Trifurcation or bifurcation lesions, $\mathrm{n}(\%)$ & $460(76.7)$ & $156(78.8)$ & 0.537 \\
\hline Chronic total occlusions, n (\%) & $141(23.5)$ & $42(21.2)$ & 0.507 \\
\hline Thrombus lesions, n (\%) & $38(6.3)$ & $7(3.5)$ & 0.139 \\
\hline Heavy calcification lesions, n (\%) & $197(32.8)$ & $68(34.3)$ & 0.696 \\
\hline Lesions > 20 mm long, $\mathrm{n}(\%)$ & $319(53.2)$ & $140(70.7)$ & $<0.001$ \\
\hline \multicolumn{4}{|l|}{ Procedural results } \\
\hline \multicolumn{4}{|l|}{ Target vessel territory } \\
\hline LM, n (\%) & $40(6.7)$ & $16(8.1)$ & 0.499 \\
\hline LAD, n (\%) & $302(50.3)$ & $98(49.5)$ & 0.838 \\
\hline LCX, n (\%) & $168(28.6)$ & $52(27.7)$ & 0.808 \\
\hline $\mathrm{RCA}, \mathrm{n}(\%)$ & $243(40.5)$ & $82(41.4)$ & 0.820 \\
\hline DES use, n (\%) & $502(83.7)$ & $164(82.8)$ & 0.783 \\
\hline BRS use, n (\%) & $27(4.5)$ & $5(2.5)$ & 0.219 \\
\hline DCB use, $\mathrm{n}(\%)$ & $34(5.7)$ & $22(11.1)$ & 0.009 \\
\hline Complete revascularization, $\mathrm{n}(\%)$ & $384(64.0)$ & $84(42.4)$ & $<0.001$ \\
\hline
\end{tabular}

Kaplan-Meier analyses revealed a significantly higher incidence of the primary endpoint (log-rank test, $P=$ 0.001; Fig. 1a) and a marginally (but non-significantly) higher incidence of the key secondary endpoint (log-rank test, $P=0.114 ;$ Fig. 1b) in patients with higher AIP values. The difference of the incidence of the 
primary endpoint was mainly driven by the increase in unplanned repeat revascularization (log-rank test, $P=0.005$; Fig. 1f) across the AIP quartiles. However, the incidence of all-cause death (log-rank test, $P=$ 0.168; Fig. 1C), CV death (log-rank test, $P=0.459$ ), non-fatal ischemic stroke (log-rank test, $P=0.167$; Fig. 1d), and non-fatal MI (log-rank test, $P=0.636$; Fig. 1e) at follow-up were similar among the AIP quartiles.

Table 5 shows univariate and multivariate Cox proportional hazards regression analyses for the primary endpoint at follow up, which includes AIP quartiles, age, BMI, PP, LVEF, SCr, LDL-C, FPG, glycosylated haemoglobin, sex, current smoking, hypertension, previous $\mathrm{MI}$, past $\mathrm{PCI}, \mathrm{PAD}$, cardiac failure, clinical presentation, coronary artery disease (CAD) severity, restenotic lesions, lesions $>20 \mathrm{~mm}$ long, use of drugcoated balloon, complete revascularization, and use of insulin at discharge. Taking Q1 as the reference, multivariate analysis showed that the AIP for Q2, Q3, and Q4 increased the HRs for the incidence of the primary endpoint (Q2: HR 1.164, 95\% Cl 0.728-1.860; Q3: HR 1.640, 95\% Cl 1.032-2.606; Q4: HR 2.153, $95 \% \mathrm{Cl} 1.355-3.421)$. Moreover, AIP used as a continuous variable was independently predictive of the primary endpoint (HR, $2.581 ; 95 \% \mathrm{Cl}, 1.499-4.444 ; P=0.001)$. Table 6 shows univariate and multivariate Cox proportional hazards regression analyses for the key secondary endpoint at follow up, which includes AIP quartiles, age, BMI, PP, LVEF, blood urea nitrogen, SCr, LDL-C, FPG, glycosylated haemoglobin, current smoking, hypertension, past PCI, PAD, cardiac failure, clinical presentation, $C A D$ severity, lesions > $20 \mathrm{~mm}$ long, complete revascularization, and use of ACEls/ARBs at discharge. Taking Q1 as the reference, multivariate analysis revealed that the AIP for Q2, Q3, and Q4 increased the HRs for the incidence of the key secondary endpoint (Q2: HR 1.063, 95\% Cl 0.397-2.849; Q3: HR 1.762, 95\% Cl 0.690-4.497; Q4: HR 2.613, 95\% Cl 1.024-6.666). Moreover, AIP used as a continuous variable was independently predictive of the key secondary endpoint $(\mathrm{HR}, 3.412 ; 95 \% \mathrm{Cl}, 1.086-10.723 ; P=0.036)$. 
Table 5

Relationship between the incidence of the primary endpoint and the AIP expressed as a categorical variable

\begin{tabular}{|c|c|c|c|c|}
\hline Variables & $\begin{array}{l}\text { Univariate analysis } \\
\mathrm{HR}(95 \% \mathrm{Cl})\end{array}$ & $P$-value & $\begin{array}{l}\text { Multivariate analysis } \\
\mathrm{HR}(95 \% \mathrm{Cl})\end{array}$ & $P$-value \\
\hline \multicolumn{5}{|l|}{ AIP quartiles } \\
\hline Q1 & Reference & & Reference & \\
\hline Q2 & $1.376(0.878-2.156)$ & 0.164 & $1.164(0.728-1.860)$ & 0.525 \\
\hline Q3 & $1.710(1.109-2.636)$ & 0.015 & $1.640(1.032-2.606)$ & 0.036 \\
\hline Q4 & $2.265(1.491-3.440)$ & $<0.001$ & $2.153(1.355-3.421)$ & 0.001 \\
\hline Age & $1.005(0.991-1.019)$ & 0.472 & $0.989(0.972-1.007)$ & 0.219 \\
\hline BMI & $0.959(0.916-1.005)$ & 0.078 & $0.931(0.884-0.982)$ & 0.008 \\
\hline PP & $1.021(1.012-1.029)$ & $<0.001$ & $1.019(1.008-1.029)$ & $<0.001$ \\
\hline LVEF & $0.974(0.959-0.990)$ & 0.002 & $0.987(0.965-1.009)$ & 0.242 \\
\hline $\mathrm{SCr}$ & $1.013(1.006-1.021)$ & 0.001 & $1.011(1.003-1.020)$ & 0.010 \\
\hline LDL-C & $1.078(0.916-1.269)$ & 0.366 & $1.021(0.842-1.239)$ & 0.832 \\
\hline FPG & $1.201(1.132-1.274)$ & $<0.001$ & $1.192(1.108-1.281)$ & $<0.001$ \\
\hline Glycosylated haemoglobin & $1.067(0.955-1.193)$ & 0.252 & $0.861(0.739-1.003)$ & 0.055 \\
\hline Male sex & $0.966(0.706-1.320)$ & 0.827 & $0.834(0.549-1.266)$ & 0.394 \\
\hline Current smoking & $1.003(0.755-1.332)$ & 0.983 & $1.256(0.874-1.805)$ & 0.219 \\
\hline Hypertension & $1.068(0.789-1.447)$ & 0.669 & $1.040(0.732-1.478)$ & 0.825 \\
\hline Previous MI & $1.492(1.095-2.033)$ & 0.011 & $0.812(0.549-1.202)$ & 0.299 \\
\hline Past PCl & $1.723(1.285-2.310)$ & $<0.001$ & $1.157(0.687-1.949)$ & 0.583 \\
\hline PAD & $2.511(1.836-3.435)$ & $<0.001$ & $1.601(1.096-2.338)$ & 0.015 \\
\hline Cardiac failure & $1.951(1.308-2.909)$ & 0.001 & $1.167(0.661-2.062)$ & 0.594 \\
\hline \multicolumn{5}{|l|}{ Clinical presentation } \\
\hline UAP & Reference & & Reference & \\
\hline NSTEMI & $0.677(0.416-1.102)$ & 0.117 & $0.531(0.310-0.909)$ & 0.021 \\
\hline STEMI & $1.098(0.690-1.747)$ & 0.695 & $1.114(0.649-1.913)$ & 0.695 \\
\hline
\end{tabular}

Abbreviations as in Tables 1 and 2. 


\begin{tabular}{|lllll|}
\hline Variables & $\begin{array}{l}\text { Univariate analysis } \\
\text { HR }(95 \% \text { Cl) }\end{array}$ & P-value & $\begin{array}{l}\text { Multivariate analysis } \\
\text { HR (95\% Cl) }\end{array}$ & $P$-value \\
\hline CAD severity & & & & \\
\hline One-vessel disease & Reference & & Reference & \\
\hline Two-vessel disease & $1.756(0.809-3.810)$ & 0.154 & $1.324(0.589-2.975)$ & 0.497 \\
\hline LM/three-vessel disease & $3.541(1.740-7.205)$ & $<0.001$ & $1.876(0.882-3.990)$ & 0.103 \\
\hline Restenotic lesions & $2.290(1.658-3.163)$ & $<0.001$ & $1.724(0.947-3.138)$ & 0.075 \\
\hline Lesions > 20 mm long & $1.984(1.461-2.695)$ & $<0.001$ & $1.574(1.132-2.189)$ & 0.007 \\
\hline DCB use & $1.913(1.228-2.980)$ & 0.004 & $1.025(0.589-1.784)$ & 0.931 \\
\hline Complete revascularization & $0.467(0.352-0.619)$ & $<0.001$ & $0.736(0.538-1.008)$ & 0.056 \\
\hline Insulin at discharge & $1.396(1.051-1.854)$ & 0.021 & $1.135(0.818-1.574)$ & 0.448 \\
\hline Abbreviations as in Tables 1 and 2. & & & \\
\hline
\end{tabular}


Table 6

Relationship between the incidence of the key secondary endpoint and the AIP expressed as a categorical variable

\begin{tabular}{|c|c|c|c|c|}
\hline Variables & $\begin{array}{l}\text { Univariate analysis } \\
\text { HR }(95 \% \mathrm{Cl})\end{array}$ & $P$-value & $\begin{array}{l}\text { Multivariate analysis } \\
\mathrm{HR}(95 \% \mathrm{Cl})\end{array}$ & $P$-value \\
\hline \multicolumn{5}{|l|}{ AIP quartiles } \\
\hline Q1 & Reference & & Reference & \\
\hline Q2 & $1.380(0.555-3.432)$ & 0.488 & $1.063(0.397-2.849)$ & 0.903 \\
\hline Q3 & $2.020(0.865-4.720)$ & 0.104 & $1.762(0.690-4.497)$ & 0.236 \\
\hline Q4 & $2.477(1.085-5.659)$ & 0.031 & $2.613(1.024-6.666)$ & 0.044 \\
\hline Age & $1.042(1.013-1.072)$ & 0.004 & $1.017(0.982-1.053)$ & 0.343 \\
\hline BMI & $0.915(0.834-1.004)$ & 0.062 & $0.844(0.751-0.948)$ & 0.004 \\
\hline PP & $1.030(1.014-1.046)$ & $<0.001$ & $1.012(0.991-1.034)$ & 0.265 \\
\hline LVEF & $0.924(0.902-0.946)$ & $<0.001$ & $0.945(0.906-0.984)$ & 0.007 \\
\hline BUN & $1.166(1.018-1.335)$ & 0.026 & $0.868(0.724-1.041)$ & 0.127 \\
\hline $\mathrm{SCr}$ & $1.025(1.014-1.036)$ & $<0.001$ & $1.016(1.001-1.030)$ & 0.031 \\
\hline LDL-C & $1.064(0.774-1.463)$ & 0.702 & $1.081(0.714-1.636)$ & 0.713 \\
\hline FPG & $1.097(0.967-1.243)$ & 0.150 & $1.017(0.880-1.176)$ & 0.818 \\
\hline Glycated haemoglobin & $1.090(0.882-1.347)$ & 0.425 & $1.220(0.932-1.597)$ & 0.148 \\
\hline Current smoking & $1.014(0.589-1.746)$ & 0.959 & $1.534(0.824-2.855)$ & 0.177 \\
\hline Hypertension & $1.333(0.725-2.450)$ & 0.354 & $1.266(0.608-2.636)$ & 0.528 \\
\hline Past PCl & $1.904(1.096-3.309)$ & 0.022 & $2.247(1.170-4.313)$ & 0.015 \\
\hline PAD & $5.804(3.402-9.902)$ & $<0.001$ & $2.888(1.545-5.399)$ & 0.001 \\
\hline Cardiac failure & $6.939(3.993-12.061)$ & $<0.001$ & $1.888(0.767-4.648)$ & 0.167 \\
\hline \multicolumn{5}{|l|}{ Clinical presentation } \\
\hline UAP & Reference & & Reference & \\
\hline NSTEMI & $1.333(0.622-2.857)$ & 0.460 & $0.501(0.199-1.258)$ & 0.141 \\
\hline STEMI & $1.920(0.896-4.115)$ & 0.094 & $1.056(0.427-2.610)$ & 0.906 \\
\hline
\end{tabular}

Abbreviations as in Tables 1 and 2. 


\begin{tabular}{|lllll|}
\hline Variables & $\begin{array}{l}\text { Univariate analysis } \\
\text { HR }(95 \% \text { Cl) }\end{array}$ & P-value & $\begin{array}{l}\text { Multivariate analysis } \\
\text { HR (95\% Cl) }\end{array}$ & $P$-value \\
\hline One-vessel disease & Reference & & Reference & \\
\hline Two-vessel disease & $0.201(0.037-1.096)$ & 0.064 & $0.059(0.009-0.402)$ & 0.004 \\
\hline LM/three-vessel disease & $1.910(0.689-5.296)$ & 0.214 & $0.418(0.121-1.444)$ & 0.168 \\
\hline Lesions $>$ 20 mm long & $2.681(1.411-5.029)$ & 0.003 & $1.828(0.882-3.789)$ & 0.105 \\
\hline Complete revascularization & $0.403(0.232-0.700)$ & 0.001 & $0.684(0.348-1.343)$ & 0.270 \\
\hline ACEls/ARBs at discharge & $2.933(1.596-5.391)$ & 0.001 & $1.782(0.883-3.599)$ & 0.107 \\
\hline Abbreviations as in Tables 1 and 2. & & & \\
\hline
\end{tabular}

Further evaluation of predictive value of AIP as a continuous variable for the primary endpoint was performed in different subgroups of the study population. Increased AIP value (per 1-unit) was consistently associated with the primary endpoint in different subgroups, including age $<60$ versus $\geq 60$ years, $\mathrm{BMI}<28$ versus $\geq 28 \mathrm{~kg} / \mathrm{m}^{2}, \mathrm{LDL}-\mathrm{C} \leq 1.8$ versus $>1.8 \mathrm{mmol} / \mathrm{L}$, female versus male, with versus without hypertension, and non-ST versus ST segment elevation ACS (Fig. 2).

From the results of the multivariate Cox proportional hazards regression analyses, we calculated the Harrell's $C$ statistic for the predictive value of the primary endpoint. The Harrell's $C$ statistic of the variables, including BMI, PP, SCr, FPG, PAD, clinical presentation, and lesions $>20 \mathrm{~mm}$ long, was 0.697 versus 0.707 after the addition of AIP quartiles; the continuous NRI was $19.1 \%(7.9-35.6 \% ; P<0.001)$.

\section{Discussion}

The main findings of the present study were as follows: (1) patients with higher AIP values had a significantly higher probability of adverse CV events by the log-rank test; (2) multivariate Cox proportional hazards analyses showed that AIP was independently and strongly associated with the primary and key secondary endpoints in T2DM and ACS patients who underwent PCl, suggesting that AIP might have a potential role in early risk stratification of such patients; (3) a significant continuous NRI showed that predictive model was improved after adding AIP to the model of other independent predictors. To the best of our knowledge, this is the first report to describe the prognostic impact of AIP in T2DM patients with ACS undergoing PCl.

Dyslipidemia plays a crucial role in the pathogenesis and progression of coronary atherosclerosis. Diabetic patients are more likely to develop coronary atherosclerosis than non-diabetic patients, which may be significantly associated with so-called diabetic dyslipidemia, which consists of elevated plasma concentrations of triglyceride-rich lipoproteins, small dense LDL-C particles and low levels of HDL-C. The different components of diabetic dyslipidemia are not isolated abnormalities but closely linked to each other metabolically [9]. Triglycerides and HDL-C are two lipid parameters measured routinely in clinical 
practice; however, neither is a consistently good proxy for plasma atherogenicity. AlP, a novel lipid index defined as $\log _{10}$ (triglycerides/HDL-C), has been demonstrated to be closely correlated with FER(HDL) and lipoprotein particle size both of which are directly involved in the pathogenesis and development of atherosclerosis, and thus AIP is regarded as an excellent indicator of atherosclerosis and can offer a benefit to identify the risk of CV disease [31].

Multiple cross-sectional studies reported that AIP was a strong predictor of CAD independent of diabetes $[23,24,26]$. Notably, the Indian Atherosclerosis Research Study revealed that addition of AIP and family history to traditional risk factors improved risk discrimination (C-index: from 0.864 to 0.873 ) in Asian Indians with CAD [19]. Intriguingly, Frohlich J, et al. found that AIP was an independent predictor of angiographically defined CAD only when FER(HDL) was omitted from multivariate analysis, which may be due to a clear internal correlation between AIP and FER(HDL) [17]. Moreover, Nam JS, et al. found that there is a significant correlation between AIP and the progression of coronary artery calcification measured by using a multi-detector computed tomography in subjects without cardiovascular disease [28]. Furthermore, in a prospective cohort study including 2676 middle-aged adults followed for 7.8 years, researchers demonstrated that the top quartiles of AIP predicted significantly age-adjusted incident CAD in both sexes, more strongly in women, after adjustment for C-reactive protein and traditional risk factors [18].

AIP has been demonstrated to be associated with mortality in elder patients and dialysis patients. Edwards MK, et al. analyzed data from the 1999-2006 National Health and Nutrition Examination Survey with follow-up through 2011 to find that AIP was positively and independently associated with mortality risk and predicted mortality risk better than individual cholesterol risk factors, among an older adult population [20]. Bendzala M, et al. also found that AIP was positively associated with the risk of all-cause death in elderly women with hypertension [21]. However, a Korean nationwide prospective cohort study including 1174 incident dialysis patients showed that AIP had a non-linear relationship with survival; both the highest and the lowest AIP quintiles were independently associated with all-cause mortality, showing a U-shaped association [22].

The predictive value of AIP has also been explored in ACS patients. Cai G, et al. retrospectively enrolled 1478 very young participants ( $\leq 35$ years of age) undergoing coronary angiography and divided them into two groups: ACS group $(n=1058)$ and non-CAD group $(n=419)$. They found that AIP was independently associated with the presence and severity of ACS in a gender-dependent manner and the prevalence of ACS, acute MI, unstable angina pectoris and the value of Gensini Score (a scoring system for evaluating CAD severity) were elevated as AIP quartiles increased [25]. Qin Z, et al. retrospectively enrolled 2356 T2DM patients who underwent PCI and followed them for 4 years. They found that AIP was an independent predictor of major cardiovascular and cerebrovascular adverse events including cardiac death, Ml, repeated revascularization, and stroke, regardless of clinical presentation [27].

AIP was reported to be positively correlated with serum malondialdehyde in menopausal women with CV disease [32]. And we know malondialdehyde can reflect the status of oxidative stress which is 
significantly associated with coronary atherosclerosis [33]. AIP was found to be associated with epicardial adipose tissue measured by using a transthoracic echocardiography or an electrocardiogramgated multidetector computed tomography [34,35]. Evidence indicates that epicardial adipose tissue directly affects coronary atherosclerosis [36]. AIP was shown to be directly and independently associated with arterial stiffness in normotensive and never-treated hypertensive subjects [37]. Increased aortic stiffness often results in early wave reflection of the aortic pulse wave which increases systolic blood pressure but decreases diastolic blood pressure, and these hemodynamic changes impair coronary perfusion, which can promote adverse CV events [30]. AIP was demonstrated to be a marker for reduced coronary flow reserve [38], and the latter was positively associated with adverse CV events. Of note, AIP includes triglycerides and HDL-C in its formula. High triglycerides and low HDL-C levels were shown to be associated with adverse CV events after ACS, independent of diabetic status $[39,40]$.

Glucose-lowering regiments including pioglitazone have been shown to be effective in reducing AIP values, thereby reducing CV risk [41, 42]. Moreover, moderate-to-vigorous physical activity, increasing aerobic exercise time, decreased sedentary behavior, and consequent high levels of cardiorespiratory fitness were reported to be inversely correlated with AIP, which implies that a healthy lifestyle helps attenuate the risk for CV disease via improvements in AIP [43-47]. As a result, use of pioglitazone and early initiation of physical activity and aerobic exercise may be important to reduce future CV risk in T2DM patients with ACS undergoing PCI who have received first-line medication therapy but still have significantly increased values of AIP.

Our study also had several important limitations. First, this was a retrospective analysis of a single centre prospective registry, which could not definitively establish causality. Second, the baseline concentrations of triglycerides and HDL-C might be affected by the use of statins before admission and glycemic control status. However, there were no significant differences among the four groups on the basis of the AIP quartiles with respect to the use of statins before admission and glycated haemoglobin which can consistently reflect glycemic control status in diabetic patients. Third, follow-up data were obtained merely via telephone; however, we confirm the authenticity of adverse events by reviewing corresponding medical records. Finally, whether the findings from the present study including only Chinese patients can be extrapolated to other ethnic groups will require further studies.

\section{Conclusions}

A higher AIP value on admission was independently and strongly associated with adverse CV events in T2DM patients with ACS undergoing PCl, suggesting that AIP might have a potential role in early risk stratification of such patients. Medical management optimization according to AIP could result in a reduced risk of subsequent $\mathrm{CV}$ events.

\section{Abbreviations}


ACEIs/ARBs: angiotensin converting enzyme inhibitors/angiotensin II receptor blockers; ACS:acute coronary syndrome; AIP:atherogenic index of plasma; BMI:body mass index; CABG:coronary artery bypass grafting; CAD:coronary artery disease; Cl:confidence interval; CV:cardiovascular; FER(HDL):cholesterol esterification rates in apoB-lipoprotein-depleted plasma; FPG:fasting plasma glucose; HDL-C:high-density lipoprotein cholesterol; HR:hazard ratio; LDL-C:low-density lipoprotein cholesterol; LVEF:left ventricular ejection fraction; MI:myocardial infarction; NRI:net reclassification improvement; PAD:peripheral artery disease; PCl:percutaneous coronary intervention; PP:pulse pressure; SCr:serum creatinine; T2DM:type 2 diabetes mellitus; TC:total cholesterol.

\section{Declarations}

\section{Conflict of interest}

The authors declare that they have no conflict of interest.

\section{Availability of data and materials}

The data, analytic methods, and study materials will not be made available to other researchers for purposes of reproducing the results or replicating the procedure.

\section{Ethics approval and consent to participate}

This study was approved by the institutional review board of Beijing Anzhen Hospital, Capital Medical University. Given the retrospective nature of this study, the requirement for informed consent was waived.

\section{Consent for publication}

Not applicable.

\section{Competing interests}

The authors declare that they have no competing interests.

\section{Funding}

This work was supported by the grant from National Key Research and Development Program of China (2017YFC0908800), Beijing Municipal Administration of Hospitals' Ascent Plan (DFL20150601) and Mission Plan (SML20180601), and Beijing Municipal Health Commission - "Project of Science and Technology Innovation Center" (PXM2019_026272_000006) (PXM2019_026272_000005). 


\section{Authors' contributions}

Xiaoteng Ma, Yan Sun, and Yujing Cheng contributed equally to this paper. All authors were involved in the conception and design of the study and in the collection, analysis, and interpretation of the data. All authors reviewed the final manuscript. All authors read and approved the final manuscript.

\section{Acknowledgements}

Not applicable.

\section{References}

1. Donahoe SM, Stewart GC, McCabe CH, Mohanavelu S, Murphy SA, Cannon CP, Antman EM. Diabetes and mortality following acute coronary syndromes. Jama. 2007;298(7):765-75.

2. Kuhl J, Jörneskog G, Wemminger M, Bengtsson M, Lundman P, Kalani M. Long-term clinical outcome in patients with acute coronary syndrome and dysglycaemia. Cardiovasc Diabetol. 2015;14:120.

3. Koshizaka M, Lopes RD, Newby LK, Clare RM, Schulte PJ, Tricoci P, Mahaffey KW, Ogawa H, Moliterno DJ, Giugliano RP, et al. Obesity, Diabetes, and Acute Coronary Syndrome: Differences Between Asians and Whites. Am J Med. 2017;130(10):1170-6.

4. Kaur R, Kaur M, Singh J. Endothelial dysfunction and platelet hyperactivity in type 2 diabetes mellitus: molecular insights and therapeutic strategies. Cardiovasc Diabetol. 2018;17(1):121.

5. Giugliano RP, Cannon CP, Blazing MA, Nicolau JC, Corbalán R, Špinar J, Park JG, White JA, Bohula EA, Braunwald E. Benefit of Adding Ezetimibe to Statin Therapy on Cardiovascular Outcomes and Safety in Patients With Versus Without Diabetes Mellitus: Results From IMPROVE-IT (Improved Reduction of Outcomes: Vytorin Efficacy International Trial). Circulation. 2018;137(15):1571-82.

6. Avogaro A, Bonora E, Consoli A, Del Prato S, Genovese S, Giorgino F. Glucose-lowering therapy and cardiovascular outcomes in patients with type 2 diabetes mellitus and acute coronary syndrome. Diab Vasc Dis Res. 2019;16(5):399-414.

7. Ahn KT, Seong SW, Choi UL, Jin SA, Kim JH, Lee JH, Choi SW, Jeong MH, Chae SC, Kim YJ, et al. Comparison of 1-year clinical outcomes between prasugrel and ticagrelor versus clopidogrel in type 2 diabetes patients with acute myocardial infarction underwent successful percutaneous coronary intervention. Med (Baltim). 2019;98(11):e14833.

8. Godoy LC, Lawler PR, Farkouh ME, Hersen B, Nicolau JC, Rao V. Urgent Revascularization Strategies in Patients With Diabetes Mellitus and Acute Coronary Syndrome. Can J Cardiol. 2019;35(8):9931001.

9. Taskinen MR, Borén J. New insights into the pathophysiology of dyslipidemia in type 2 diabetes. Atherosclerosis. 2015;239(2):483-95. 
10. Dobiásová M, Frohlich J. [The new atherogenic plasma index reflects the triglyceride and HDLcholesterol ratio, the lipoprotein particle size and the cholesterol esterification rate: changes during lipanor therapy]. Vnitr Lek. 2000;46(3):152-6.

11. Dobiásová $M$, Frohlich $J$. The plasma parameter log (TG/HDL-C) as an atherogenic index: correlation with lipoprotein particle size and esterification rate in apoB-lipoprotein-depleted plasma (FER(HDL)). Clin Biochem. 2001;34(7):583-8.

12. Dobiásová $M$, Raslová $K$, Rauchová $H$, Vohnout $B$, Ptácková $K$, Frohlich J. Atherogenic lipoprotein profile in families with and without history of early myocardial infarction. Physiol Res. 2001;50(1):18.

13. Dobiásová $M$, Urbanová Z, Samánek M. Relations between particle size of HDL and LDL lipoproteins and cholesterol esterification rate. Physiol Res. 2005;54(2):159-65.

14. Rašlová $K$, Dobiášová $M$, Hubáček JA, Bencová $D$, Siváková $D$, Danková $Z$, Franeková J, Jabor $A$, Gašparovič J, Vohnout B. Association of metabolic and genetic factors with cholesterol esterification rate in HDL plasma and atherogenic index of plasma in a 40 years old Slovak population. Physiol Res. 2011;60(5):785-95.

15. Dobiásová M, Frohlich J, Sedová M, Cheung MC, Brown BG. Cholesterol esterification and atherogenic index of plasma correlate with lipoprotein size and findings on coronary angiography. $J$ Lipid Res. 2011;52(3):566-71.

16. Quispe R, Manalac RJ, Faridi KF, Blaha MJ, Toth PP, Kulkarni KR, Nasir K, Virani SS, Banach M, Blumenthal RS, et al. Relationship of the triglyceride to high-density lipoprotein cholesterol (TG/HDLC) ratio to the remainder of the lipid profile: The Very Large Database of Lipids-4 (VLDL-4) study. Atherosclerosis. 2015;242(1):243-50.

17. Frohlich J, Dobiásová M. Fractional esterification rate of cholesterol and ratio of triglycerides to HDLcholesterol are powerful predictors of positive findings on coronary angiography. Clin Chem. 2003;49(11):1873-80.

18. Onat A, Can G, Kaya H, Hergenç G. "Atherogenic index of plasma" (log10 triglyceride/high-density lipoprotein-cholesterol) predicts high blood pressure, diabetes, and vascular events. J Clin Lipidol. 2010;4(2):89-98.

19. Shanker J, Kakkar VV. Contribution of classical and emerging risk factors to coronary artery disease in Asian Indians. Int J Cardiol. 2016;214:97-106.

20. Edwards MK, Blaha MJ, Loprinzi PD. Atherogenic Index of Plasma and Triglyceride/High-Density Lipoprotein Cholesterol Ratio Predict Mortality Risk Better Than Individual Cholesterol Risk Factors, Among an Older Adult Population. Mayo Clin Proc. 2017;92(4):680-1.

21. Bendzala M, Sabaka P, Caprnda M, Komornikova A, Bisahova M, Baneszova R, Petrovic D, Prosecky $\mathrm{R}$, Rodrigo $\mathrm{L}$, Kruzliak $\mathrm{P}$, et al. Atherogenic index of plasma is positively associated with the risk of allcause death in elderly women: A 10-year follow-up. Wien Klin Wochenschr. 2017;129(21-22):793-8.

22. Lee MJ, Park JT, Han SH, Kim YL, Kim YS, Yang CW, Kim NH, Kang SW, Kim HJ, Yoo TH. The atherogenic index of plasma and the risk of mortality in incident dialysis patients: Results from a 
nationwide prospective cohort in Korea. PLoS One. 2017;12(5):e0177499.

23. Cai G, Shi G, Xue S, Lu W. The atherogenic index of plasma is a strong and independent predictor for coronary artery disease in the Chinese Han population. Med (Baltim). 2017;96(37):e8058.

24. Wu TT, Gao Y, Zheng YY, Ma YT, Xie X. Atherogenic index of plasma (AIP): a novel predictive indicator for the coronary artery disease in postmenopausal women. Lipids Health Dis. 2018;17(1):197.

25. Cai G, Liu W, Lv S, Wang X, Guo Y, Yan Z, Du Y, Zhou Y. Gender-specific associations between atherogenic index of plasma and the presence and severity of acute coronary syndrome in very young adults: a hospital-based observational study. Lipids Health Dis. 2019;18(1):99.

26. Guo Q, Zhou S, Feng X, Yang J, Qiao J, Zhao Y, Shi D, Zhou Y. The sensibility of the new blood lipid indicator-atherogenic index of plasma (AIP) in menopausal women with coronary artery disease. Lipids Health Dis. 2020;19(1):27.

27. Qin Z, Zhou K, Li Y, Cheng W, Wang Z, Wang J, Gao F, Yang L, Xu Y, Wu Y, et al. The atherogenic index of plasma plays an important role in predicting the prognosis of type 2 diabetic subjects undergoing percutaneous coronary intervention: results from an observational cohort study in China. Cardiovasc Diabetol. 2020;19(1):23.

28. Nam JS, Kim MK, Nam JY, Park K, Kang S, Ahn CW, Park JS. Association between atherogenic index of plasma and coronary artery calcification progression in Korean adults. Lipids Health Dis. 2020;19(1):157.

29. Ma X, Dong L, Shao Q, Cheng Y, Lv S, Sun Y, Shen H, Wang Z, Zhou Y, Liu X. Triglyceride glucose index for predicting cardiovascular outcomes after percutaneous coronary intervention in patients with type 2 diabetes mellitus and acute coronary syndrome. Cardiovasc Diabetol. 2020;19(1):31.

30. Ma X, Dong L, Shao Q, Zhou Z, Tian J, Ma Y, Yang J, Lv S, Cheng Y, Shen H, et al. Predictive performance of aortic arch calcification for clinical outcomes in patients with acute coronary syndrome that undergo percutaneous coronary intervention: A prospective clinical study. Med (Baltim). 2019;98(48):e18187.

31. Dobiášová M. Atherogenic impact of lecithin-cholesterol acyltransferase and its relation to cholesterol esterification rate in HDL (FER(HDL)) and AIP [log(TG/HDL-C)] biomarkers: the butterfly effect? Physiol Res. 2017;66(2):193-203.

32. Amrita J, Mahajan M, Bhanwer AJ, Mohan G: Oxidative Stress: An Effective Prognostic Tool for an Early Detection of Cardiovascular Disease in Menopausal Women. Biochem Res Int 2016, 2016:6157605.

33. Haberland ME, Fong D, Cheng L. Malondialdehyde, modified lipoproteins, and atherosclerosis. Eur Heart J. 1990;11(Suppl E):100-4.

34. Erdur MF, Tonbul HZ, Ozbiner H, Ozcicek A, Ozcicek F, Akbas EM, Ozbek O, Hamur H, Turkmen K. The relationship between atherogenic index of plasma and epicardial adipose tissue in hemodialysis and peritoneal dialysis patients. Ren Fail. 2013;35(9):1193-8.

35. Akbas EM, Hamur H, Demirtas L, Bakirci EM, Ozcicek A, Ozcicek F, Kuyrukluyildiz U, Turkmen K. Predictors of epicardial adipose tissue in patients with type 2 diabetes mellitus. Diabetol Metab 
Syndr. 2014;6:55.

36. Madonna R, Massaro M, Scoditti E, Pescetelli I, De Caterina R. The epicardial adipose tissue and the coronary arteries: dangerous liaisons. Cardiovasc Res. 2019;115(6):1013-25.

37. Choudhary MK, Eräranta A, Koskela J, Tikkakoski AJ, Nevalainen PI, Kähönen M, Mustonen J, Pörsti I. Atherogenic index of plasma is related to arterial stiffness but not to blood pressure in normotensive and never-treated hypertensive subjects. Blood Press. 2019;28(3):157-67.

38. Kul Ş, Çalışkan Z, Güvenç TS, Güvenç R, Çalışkan M. Plasma lipids in patients with inflammatory bowel disease: Observations on the associations between lipid indices and coronary flow reserve. Wien Klin Wochenschr. 2020;132(11-12):283-94.

39. Schwartz GG, Abt M, Bao W, DeMicco D, Kallend D, Miller M, Mundl H, Olsson AG. Fasting triglycerides predict recurrent ischemic events in patients with acute coronary syndrome treated with statins. J Am Coll Cardiol. 2015;65(21):2267-75.

40. Nakazawa M, Arashi H, Yamaguchi J, Ogawa H, Hagiwara N. Lower levels of high-density lipoprotein cholesterol are associated with increased cardiovascular events in patients with acute coronary syndrome. Atherosclerosis. 2020;303:21-8.

41. Rodríguez A, Reviriego J, Karamanos V, del Cañizo FJ, Vlachogiannis N, Drossinos V. Management of cardiovascular risk factors with pioglitazone combination therapies in type 2 diabetes: an observational cohort study. Cardiovasc Diabetol. 2011;10:18.

42. Tan MH, Johns D, Glazer NB. Pioglitazone reduces atherogenic index of plasma in patients with type 2 diabetes. Clin Chem. 2004;50(7):1184-8.

43. Edwards MK, Blaha MJ, Loprinzi PD. Influence of sedentary behavior, physical activity, and cardiorespiratory fitness on the atherogenic index of plasma. J Clin Lipidol. 2017;11(1):119-25.

44. Shen S, Lu Y, Dang Y, Qi H, Shen Z, Wu L, Li F, Yang C, Qiang D, Yang Y, et al. Effect of aerobic exercise on the atherogenic index of plasma in middle-aged Chinese men with various body weights. Int $\mathrm{J}$ Cardiol. 2017;230:1-5.

45. Shen S, Qi H, He X, Lu Y, Yang C, Li F, Wang L, Qiang D, Shui K, Zhou L, et al. Aerobic Exercise for a Duration of 90 min or Longer Per Week may Reduce the Atherogenic Index of Plasma. Sci Rep. 2018;8(1):1730.

46. Edwards M, Loprinzi P. The Dose-Response Association Between Reported Moderate to Vigorous Intensity Physical Activity and Atherogenic Index of Plasma: NHANES, 1999-2006. J Phys Act Health. 2019;16(5):368-70.

47. Reyes-Ferrada W, Solis-Urra P, Plaza-Díaz J, Sadarangani KP, de Moraes Ferrari GL, RodríguezRodríguez F, Cristi-Montero C. Cardiorespiratory Fitness, Physical Activity, Sedentary Time and Its Association with the Atherogenic Index of Plasma in Chilean Adults: Influence of the Waist Circumference to Height Ratio. Nutrients 2020, 12(5).

\section{Figures}




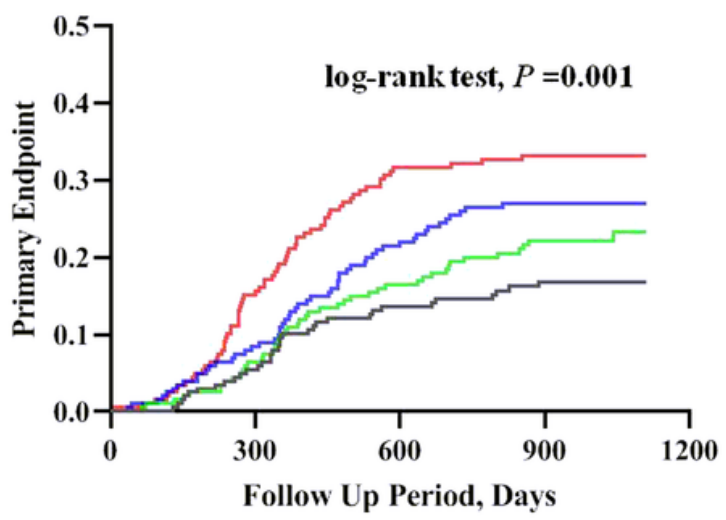

a

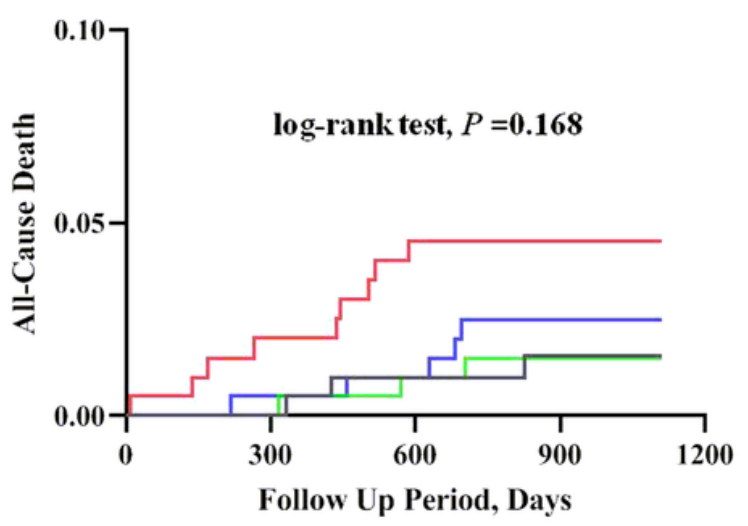

c

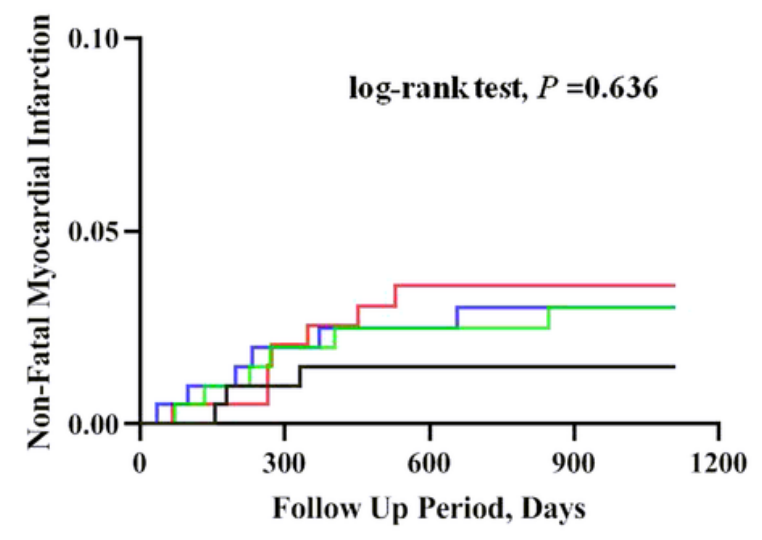

e
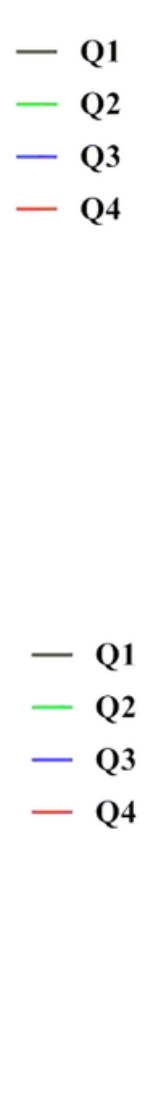

- Q1
- Q2
- Q3
- Q4

\section{Figure 1}

Kaplan-Meier curves of the incidences of the primary and key secondary endpoints and each component event of the primary endpoint for the AIP quartiles. The primary endpoint was defined as a composite of all-cause death, non-fatal ischemic stroke, non-fatal myocardial infarction, and unplanned repeat revascularization. The key secondary endpoint was a composite of CV death, non-fatal ischemic stroke, or non-fatal myocardial infarction.

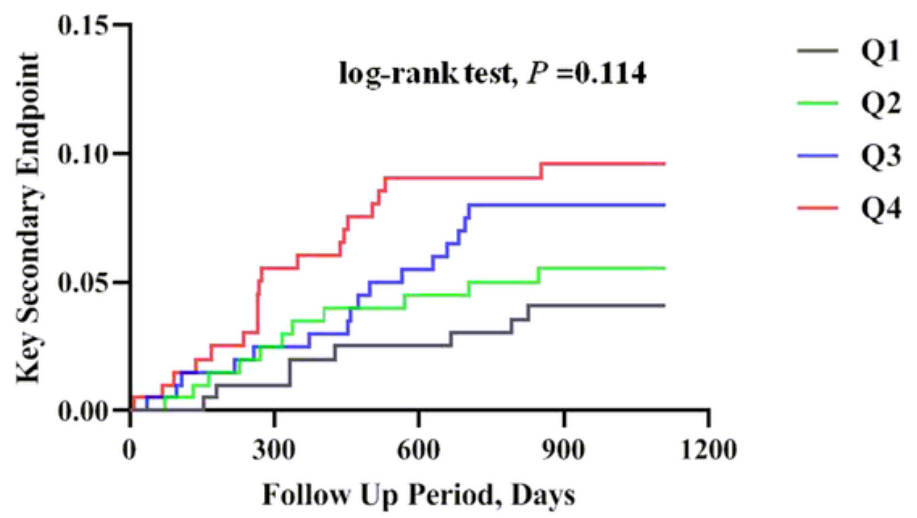

b

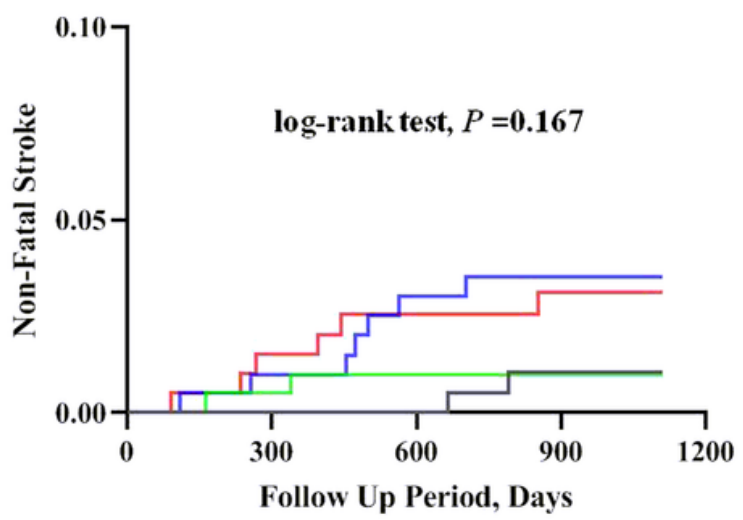

d

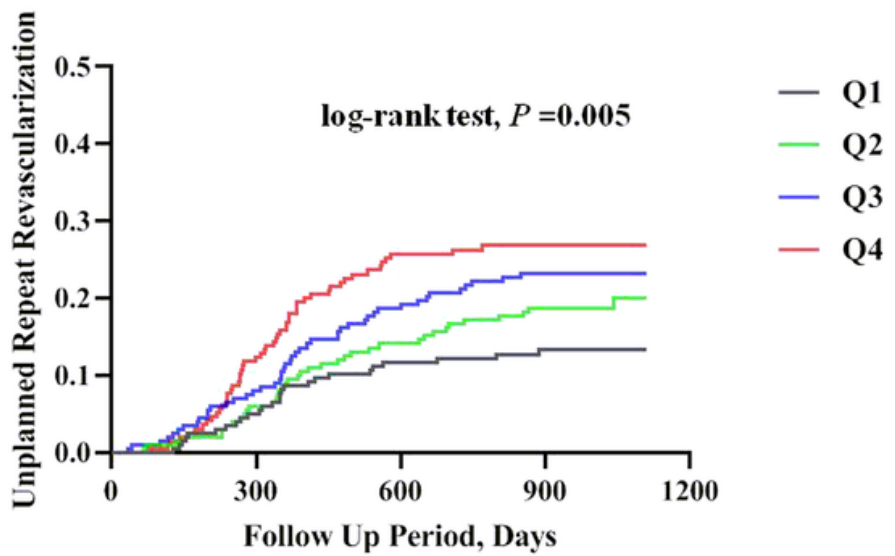

f 
HR $(95 \% \mathrm{CI})$ Per 1-unit increase in AIP 2.926(1.478-5.793)

2.969 (1.415-6.228)

$2.539(1.439-4.478)$

5.224 (1.851-14.739)

2.615 (1.501-4.556)

$5.366(1.957-14.717)$

4.626(1.602-13.357)

2.402 (1.373-4.204)

2.597 (1.083-6.223)

3.047 (1.707-5.440)

$2.593(1.553-4.330)$

$7.634(1.712-34.045)$
$P$ for interaction

0.977

0.194

0.218

0.325

0.771

0.226

ST segment elevation ACS

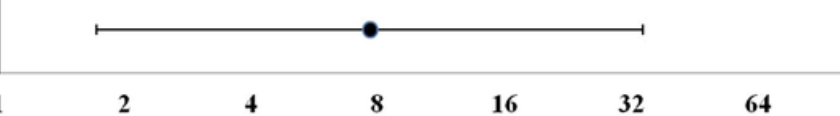

\section{Figure 2}

Cox proportional hazards analyses for the primary endpoint at follow up in different subgroups. HR was evaluated by per 1-unit increase in AIP. BMI indicates body mass index; LDL-C, low-density lipoprotein cholesterol; ACS acute coronary syndrome; HR, hazard ratio; 95\% Cl, 95\% confidence interval. 